\title{
Effect of CB1 Receptor Blockade on Food-Reinforced Responding and Associated Nucleus Accumbens Neuronal Activity in Rats
}

\author{
Giovanni Hernandez and Joseph F. Cheer \\ Departments of Anatomy and Neurobiology, and Psychiatry, University of Maryland School of Medicine, Baltimore, Maryland 21201
}

\begin{abstract}
Studies have shown that disruption of cannabinoid receptor signaling reduces operant responses for rewards; yet it is unknown whether changes in neural activity at dopamine terminal regions such as the nucleus accumbens (NAc) underlie these behavioral effects. To study the neural correlates that accompany the disruption of endogenous cannabinoid (eCB) signaling in a food-motivated task, we recorded the neural activity and local field potentials (LFPs) from the NAc. A within-subject design was used for recordings as rats engaged in lever-pressing behavior for sucrose chocolate-flavored pellets delivered during responding in a progressive ratio (PR) schedule of reinforcement. Rats were food restricted to $85 \pm 5 \%$ of their free body weight and trained under a PR until a stable breakpoint was observed ( 12 sessions \pm 3 ). Once performance was stable, recordings were made under baseline, vehicle, and following administration of the cannabinoid inverse agonist rimonabant $(150 \mu \mathrm{g} / \mathrm{kg}$, i.v). NAc neurons encoded reward-predictive cues as well as food reward delivery. Rimonabant administration robustly reduced breakpoints in all rats tested, as previously reported. We found that this reduction is accompanied by a profound attenuation in the strength and coordination of specific event-related spiking activity. Moreover, rimonabant decreased LFP gamma power at $80 \mathrm{~Hz}$ (high gamma) at reward delivery and gamma power at $50 \mathrm{~Hz}$ (low gamma) at cue onset. Together the present results indicate that the eCB system sculpts neural activity patterns that accompany PR performance and reward consumption.
\end{abstract}

\section{Introduction}

Endogenous cannabinoids (eCBs) and in particular CB1 receptors (CB1Rs) play a key role in the modulation of reinforcement processing (Robbe et al., 2002, Cota et al., 2003; Le Foll and Goldberg, 2005; Melis et al., 2004; Oleson et al., 2012). eCBs are released "on demand" and act in a retrograde manner. They activate CB1Rs located presynaptically on excitatory and inhibitory terminals (Alger, 2002; Wilson and Nicoll, 2002). CB1Rs negatively modulate transduction pathways through coupling to $G$ proteins. Their activation initiates several transduction mechanisms including the activation of potassium channels and of MAP kinase, as well as the inhibition of voltage-dependent calcium channels and of adenylyl cyclase, leading to a decrease in the probability of neurotransmitter release (Bidaut-Russell et al., 1990; Henry and Chavkin, 1995; Twitchell et al., 1997; Hoffman and Lupica, 2000). These mechanisms play a regulatory role in

Received April 16, 2012; revised June 28, 2012; accepted July 8, 2012.

Author contributions: G.H. and J.F.C. designed research; G.H. performed research; J.F.C. contributed unpublished reagents/analytic tools; G.H. analyzed data; G.H. and J.F.C. wrote the paper.

This research was supported by National Institutes of Health Grants DA022340 and DA025890 (J.F.C.) and a Le Fonds de recherche du Québec - Nature et technologies fellowship (G.H.). We thank Mike Beckert for expert technical assistance and Drs. Joshua Berke, David Redish, Matthijs van der Meer, Kuei Tseng, and Carien Lansink for valuable comments.

The authors declare no competing financial interests.

Correspondence should be addressed to Joseph F. Cheer or Dr. Giovanni Hernandez, University of Maryland School of Medicine, Department of Anatomy and Neurobiology, 20 Penn Street, Baltimore, MD 21201. E-mail: jchee001@umaryland.edu or giovannih@gmail.com.

DOI:10.1523/JNEUROSCI.1833-12.2012

Copyright $\odot 2012$ the authors $\quad 0270-6474 / 12 / 3211467-11 \$ 15.00 / 0$ the excitability of neurons located in several nuclei of brain reward pathways including those that project to the ventral tegmental area (VTA) and the nucleus accumbens (NAc) (Domenici et al., 2006, Oleson et al., 2012). Activation of CB1Rs in the VTA modulates dopamine (DA) neuronal activity (Cheer et al., 2000, 2003; Szabo et al., 2002; Lupica and Riegel, 2005), as well as drug and cue-evoked DA release in the NAc (Cheer et al., 2007; Li et al., 2009, Oleson et al., 2012).

At the behavioral level, the modulation of reinforcement processing by eCBs has been demonstrated using multiple behavioral paradigms and rewards (Valjent et al., 2002; De Vry et al., 2004; Soria et al., 2005, Filip et al., 2006; Shoaib, 2008). A schedule of reinforcement that has been consistently used is the progressive ratio (PR); here, the requirement to obtain a single reward is exponentially increased within a single session until responding ceases. The ratio at which this occurs is called "breakpoint" (Hodos, 1961). When exogenous as well as eCB agonists are tested in this schedule, increased breakpoints are observed (Gallate et al., 1999; Higgs et al., 2005; Solinas et al., 2005; Ward and Dykstra, 2005, Oleson et al., 2012), whereas CB1R antagonists have the opposite effect (Solinas and Goldberg, 2005; Ward and Dykstra, 2005; Rasmussen and Huskinson, 2008; Xi et al., 2008).

Despite abundant evidence supporting the role of CB1Rs in the modulation of reward processing as well as electrophysiological evidence that manipulation of CB1Rs alters neural activity in different reward circuit nuclei, little is known regarding how these behavioral changes accompany real-time neural activity of the NAc, a limbic-motor interface (Mogenson et al., 1980). Dif- 


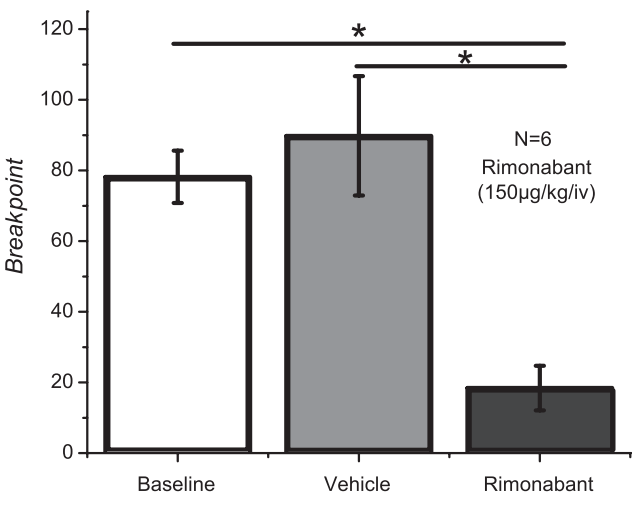

Figure 1. Rimonabant significantly reduces breakpoints obtained in a PR schedule $(N=6)$.
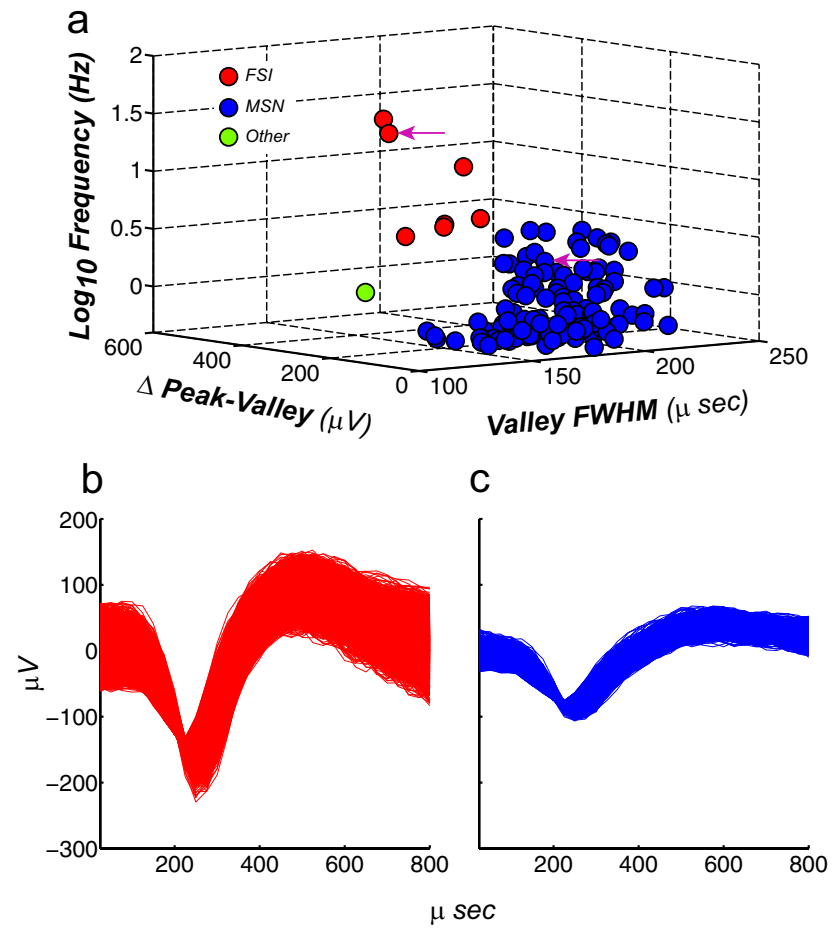

Figure 2. Sorting criteria for identifying putative MSNs and FSIs. The total of number neurons recorded was 133. $\boldsymbol{a}$, Criteria used for identifying putative neuron types were firing rate ( $z$-axis), amplitude ( $x$-axis), and valley FWHM ( $y$-axis). Using these criteria a total of 125 neurons were identified as putative MSN, 7 as putative $F S I$, and 1 as other type. Arrowheads denote selected waveforms depicted in (b) putative FSI and (c) putative MSN.

\section{Table 1. Average and SEM of the criteria used to identify MSN and FSIs}

\begin{tabular}{lrr}
\hline Putative neuron classification & \multicolumn{1}{c}{ FSI } & \multicolumn{1}{c}{ MSN } \\
\hline Firing rate Hz (mean \pm SEM) & $16.72( \pm 2.49)$ & $1.12( \pm 0.01)$ \\
Valley ms (FWHM) & $133.71( \pm 2.14)$ & $179.71( \pm 0.16)$ \\
Peak-valley distance $(\mathrm{mV})$ & $147.62( \pm 2.07)$ & $103.00( \pm 0.46)$
\end{tabular}

ferent lines of evidence point toward specific roles played by NAc neurons in different aspects of reward processing (Cardinal et al., 2002; Berridge, 2007) and motivational control of behavior (Salamone and Correa, 2002). These processes are assumed to bias behavioral output and action selection toward the highest immediate subjective value (Nicola et al., 2004a; Samejima et al., 2005; Kim et al., 2009). Here we identified electrophysiological changes that occur in the NAc of rats engaged in a PR schedule, using food as a reward, while eCB signaling was pharmacologically impeded using a CB1R antagonist.

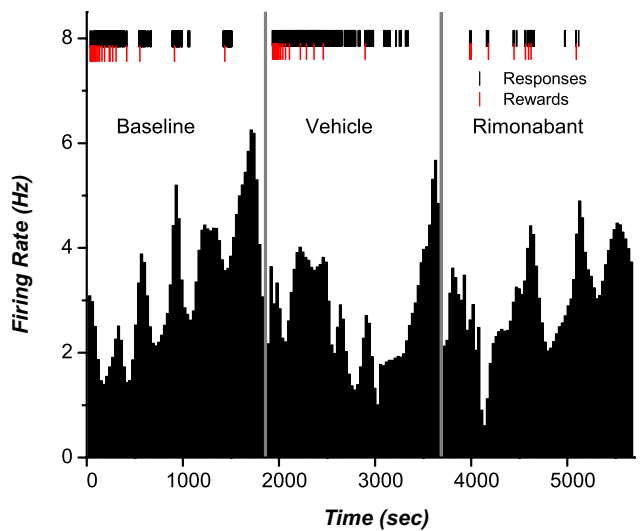

Figure 3. Rate histograms showing an LPE neuron. Black tick marks show lever presses and red ticks indicate reward delivery. Vehicle and rimonabant were injected $1 \mathrm{~min}$ before the start of the schedule and neural recording (represented by the vertical gray lines). The highest firing rate peak is observed just after reaching the breakpoint.

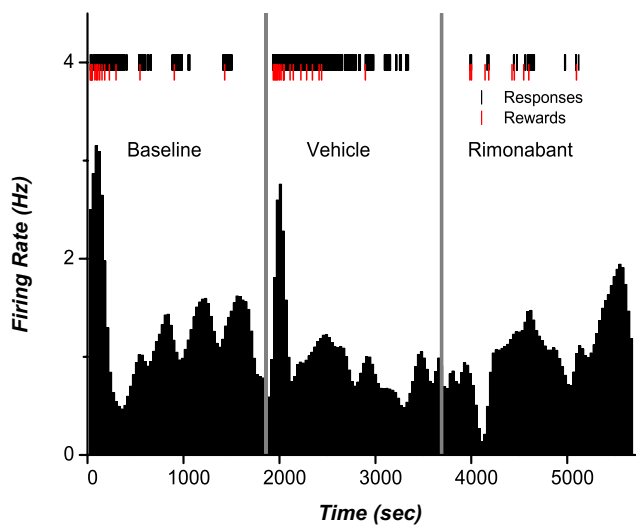

Figure 4. Rate histograms showing a typical LPI neuron. Black tick marks show lever presses and red ticks indicate reward delivery. Vehicle and rimonabant were injected $1 \mathrm{~min}$ before the start of the schedule and neural recording (represented by the vertical gray lines). The highest firing rate peak is observed just at the beginning of the session.

Table 2. Number and percentage of NAc neurons exhibiting different types of patterned discharges during reward delivery and cue onset

\begin{tabular}{lrrrrr}
\hline & \multicolumn{2}{c}{ Reward delivery } & & \multicolumn{2}{c}{ Trial start } \\
\cline { 2 - 3 } Firing pattern & \multicolumn{1}{c}{$n$} & & & \multicolumn{1}{c}{$n$} \\
\hline Excitation at 0 & 27.00 & 20.30 & & 10.00 & 7.50 \\
Long excitation after 0 & 5.00 & 3.75 & & 4.00 & 3.00 \\
Inhibition at 0 & 0.00 & 0.00 & & 0.00 & 0.00 \\
Long inhibition after 0 & 4.00 & 3.00 & & 5.00 & 3.75 \\
Total & 36.00 & 27.05 & & 19.00 & 14.25 \\
\hline
\end{tabular}

\section{Materials and Methods}

Subjects and surgery. Six (6) male Sprague Dawley rats (Charles River Laboratories) with indwelling jugular vein catheters were used. Rats were individually housed in a temperature- and humidity-controlled room with a $12 \mathrm{~h}$ light/dark cycle (lights on at 7:00). Animals were stereotaxically implanted, under isoflurane anesthesia, with two 16 microwire arrays of electrodes made of Teflon-insulated stainless steel $(0.25 \mathrm{~mm}$ interelectrode space, $0.5 \mathrm{~mm}$ inter-row space; Micro Probe). The arrays were aimed at the NAc with the center of each array lowered at the following coordinates from bregma (1.7 anteroposterior, 1.5 mediolateral, and -8.0 dorsoventral from dura). Electrodes were fixed to the skull with acrylic cement secured with stainless steel bone screws. A stainless steel wire from each array served as a ground electrode and was inserted caudal to the arrays in the midbrain/cerebellum interface (a region where 

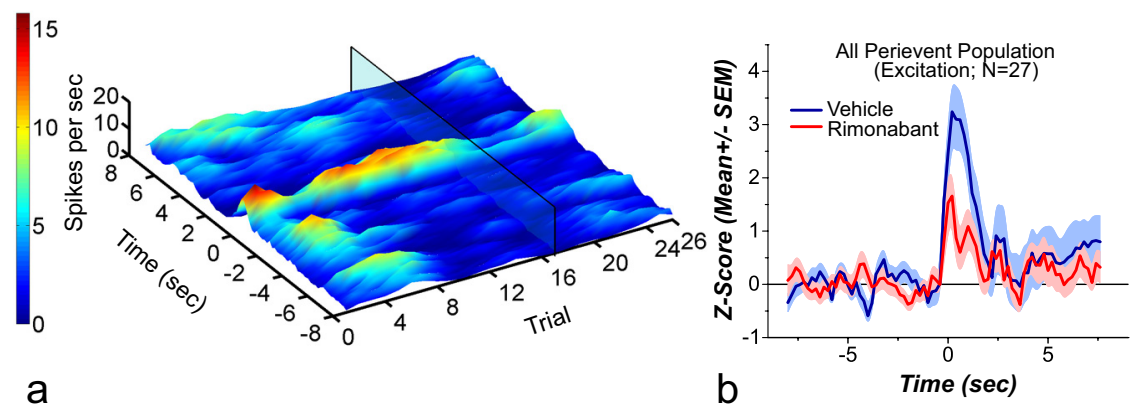

a

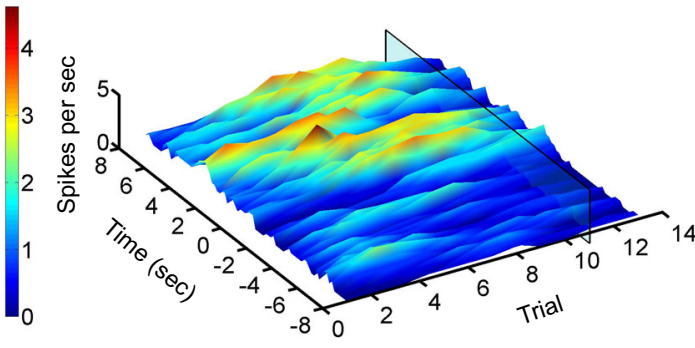

C

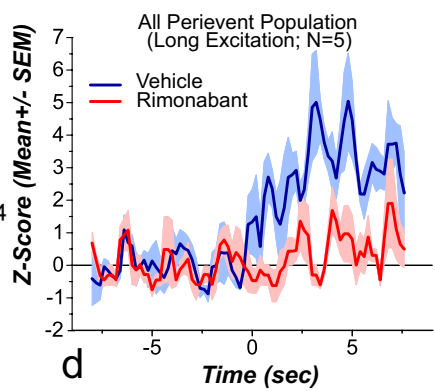

5
4
3
3
2
1
0

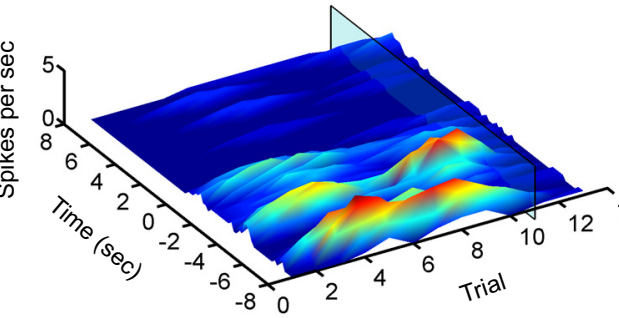

e

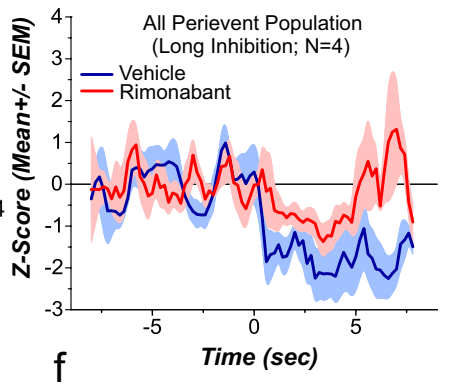

Figure 5. 3D rasters and normalized average from neurons responsive to reward delivery. The 3D perievent rasters are aligned to reward delivery at time 0 ; rimonabant administration is denoted by the translucent rectangle. $\boldsymbol{a}$, Shows a representative neuron that presented a transient increase in firing rate after reward delivery and its associated cues. Rimonabant administration reduced patterned spiking activity. $\boldsymbol{b}$, Shows the mean normalized firing rate from all the neurons showing a transient increase in firing rate $(n=27)$. Rimonabant produces a significant reduction in the firing rate peak observed after reward delivery. $c$, Shows a representative neuron that presented a sustained increase in firing rate after reward delivery. Rimonabant administration reduced this increase. $\boldsymbol{d}$, Shows the mean normalized firing rate from all the neurons showing a sustained increase in firing rate $(n=5)$. Rimonabant produces a significant reduction in the firing increase observed after reward delivery. $\boldsymbol{e}$, Shows a representative neuron that presented a sustained decrease in firing rate after reward delivery. Rimonabant administration reversed this inhibitory pattern. $\boldsymbol{f}$, Shows the mean normalized firing rate from all the neurons showing a sustained decrease in firing rate $(n=4)$. Rimonabant produced a significant change in the magnitude of the inhibition. Following rimonabant administration the decrease observed after reward delivery failed to reach statistical significance.

gamma oscillations were not observed). Rats were allowed to recover for at least $10 \mathrm{~d}$, during which time they received food and water ad libitum. After that period rats were food restricted at $85 \pm 5 \%$ of their free-feeding weight before initial training and were maintained around this weight throughout the experiment. All procedures were performed in accordance with established practices as described in the National Institutes of Health Guide for Care and Use of Laboratory Animals. In addition, all procedures were reviewed and approved by the Animal Care and Use Committee of University of Maryland School of Medicine.

Drugs. SR141716A (rimonabant) was provided by the National Institute on Drug Abuse Drug Supply Program (Research Triangle Park, Raleigh, NC) and dissolved in a solution of (1:1:18) ethanol, Emulphor (Rhodia), and saline and injected intravenously at 0 and $150 \mu \mathrm{g} / \mathrm{kg}$. This dose is selected for its inability to alter locomotor activity (Cippitelli et al., 2005).
Behavioral training. Experiments were conducted in rat operant conditioning chambers (12.5 L $\times 13.5 \mathrm{~W} \times 13.5$ ” $\mathrm{H}$; Med Associates) located within ventilated sound attenuation chambers. The operant boxes were equipped with a house light, two cue lights above two retractable levers (Coulbourn Instruments), a modular pellet dispenser and receptacle, a sonoalert module that delivers a $2900 \mathrm{~Hz}$ tone, and a white noise amplifier. Rats were initially trained under a fixed-ratio 1 (FR1) with an intertrial interval of $10 \mathrm{~s}$. During the trials and throughout the experiment both retractable levers were present, but only one was associated with an illuminated cue light and reward delivery (active lever). Responses on the other lever (inactive lever) were recorded but did not have any scheduled consequences. A trial began with the cue light on top of the active lever, the house light on, and the extension of the active and inactive levers. Once the rat pressed down the active lever both levers retracted and a 45 mg sucrose chocolate-flavored pellet (BioServ) was delivered, cue light and house light extinguished, and a $2900 \mathrm{~Hz}$ tone started; these stimuli will be referred as reward associated cues. At the end of the $10 \mathrm{~s}$ intertrial interval, the tone was turned off and a new trial began. Once rats reliably pressed the lever on 3 consecutive days (earned $>280$ pellets in a $1 \mathrm{~h}$ session) they were considered lever trained. A PR schedule was then implemented in which the number of responses required to obtain a food pellet increased for successive reinforcers. The progression was derived from the equation: response ratio $=\left[5 \times \mathrm{e}^{(0.2 \times \text { reinforcer number })}-\right.$ 5], and yielded ratios of $1,2,4,6,9,12,15,20$, $25,32,40,50,62,77,95,118$, etc., following rounding to the closest integer. A trial was defined by the start of a new response ratio and it ended when the reward was delivered upon completion of the response ratio schedule in effect. As with the FR1 schedule a trial began with the cue light on top of the active lever, the house light on, and the extension of the active and inactive levers. Once the reward was delivered, the cue light and house light were extinguished and a $2900 \mathrm{~Hz}$ tone started. At the end of the $10 \mathrm{~s}$ intertrial interval, the tone was turned off and a new trial began. Sessions lasted until a period of $20 \mathrm{~min}$ had elapsed without earning a food pellet. The number of food pellets earned before each respective breakpoint was defined as the highest completed ratio, and the total number of responses made was recorded. Testing began when breakpoints did not vary by $>1$ ratio under or over the previous session for at least three sessions. Once rats had stable breakpoints, sequential PR schedules were run on a single day. The rats were treated with vehicle and rimonabant $(150 \mu \mathrm{g} / \mathrm{kg}$, i.v.) injected 1 min before the recording of the neural activity of the corresponding condition and the start of the schedule.

Electrophysiological experiments. Neural activity was recorded using commercially available hardware and software, including headstage and programmable amplifiers, filters, and multichannel spike-sorting software (Plexon Instruments). Discrimination of individual units was performed off-line using principal component analysis of waveform shape, followed by manual confirmation of sorting validity. Signals were routed to a differential preamplifier (fixed $50 \times$ gain; Plexon) and relayed to a Multineuron Acquisition Processor (Plexon), which allows 
for computer-controlled, channel-specific signal amplification (gain steps 1-30, total gain $1000-32,000 \times$ ), filtering (second-order 500 $\mathrm{Hz}$ low cut, $5 \mathrm{kHz}$ high cut), and analog to digital conversion (32 simultaneous sampling 12-bit converters, $75 \mathrm{kHz}$ ). Single units were identified by constancy of waveform shape, autocorrelograms, and interspike interval. The spiking frequency, amplitude of the spikes as the maximal peak-valley difference, and the full-width at half-maximum (FWHM) for valley were used to help in the classification between fast-spiking interneurons (FSI) and medium spiny neurons (MSN) (Burkhardt et al., 2009).

Cross-correlograms were constructed using 160 bins (bin width: $0.005 \mathrm{~s}$ ) within a time range of $\pm 0.4 \mathrm{~s}$ (erected using the same number of spikes per condition for bias correction). Peaks and troughs in cross-correlation histograms were initially identified through visual inspection and then confirmed by using $Z$ scores of the peaks and troughs; the data contained $0.1 \mathrm{~s}$ before histogram shoulder was used for the background calculation. Those peaks and troughs with $Z$ scores above/below $2.58 /-2.58(p=0.01)$ were selected for analysis as a reliable indicator that a nonflat crosscorrelation existed.

LFPs were recorded simultaneously with single unit activity from the same microwire arrays and acquired at $1 \mathrm{kHz}$. All raw LFP voltage traces and whole-session power spectral densities (PSDs) were visually inspected to confirm recording quality before further analysis. Sorted files were analyzed using NeuroExplorer (Plexon Instruments) and custom-developed MATLAB (MathWorks) code. Neural activity was initially characterized via perievent rasters. A change in firing rate was considered significant if the neural response changed $>2$ SDs from baseline firing rate. For LFP analysis, the multitaper method was used to estimate frequency spectra (Pesaran et al., 2002). Spectrograms were constructed by plotting spectral power during a series of overlapping constant-width time windows. The data contained $2 \mathrm{~s}$ before the peak or trough shoulder was used for $Z$ score background calculations. Spikefield coherence was performed using the Sigtool routine (Lidierth, 2009), a bootstrapping sampling procedure (Efron, 1979) implemented in MATLAB, to generate 1000 samples with replacement of the spike-field coherence values and compute $95 \%$ confidence intervals. Figures were prepared in Origin (OriginLab) or MATLAB.

Data analysis. Repeated-measures ANOVA was used to determine statistical significance for the change in breakpoint, and Tukey's HSD post hoc test was used to evaluate any difference between the treatments. The firing rate of each neuron was normalized using $Z$ scores, in which the firing rate of $7 \mathrm{~s}$ before the event of interest was used to obtain the average and SD of the firing rate. The normalized data were used for the statistical analysis of the neuronal firing rates.

To classify neurons as lever press excited (LPE) or lever press inhibited (LPI) using integrated firing rate histograms following Nicola and Deadwyler's (2000) classification, we divided the firing rate and lever press responses in $180 \mathrm{~s}$ windows and for each lever press bout or pause, the average firing rate at that window was contrasted against the previous one so that the firing rate could be classified as increased or reduced. This produced a $2 \times 2$ table for which a $\chi^{2}$ test was calculated. Significant $\chi^{2}$ values $(p \leq 0.05)$ were taken as an indication of a nonrandom distribution between the variables and on those tables the $\varphi$ coefficient of association was calculated. Neurons with a positive coefficient were classified as LPE, whereas those with a negative coefficient were classified as LPI.

For perievent raster and local field potential analyses paired Student's $t$ tests were used to contrast peaks and troughs against vehicle and baseline conditions. All statistical tests were calculated using Statistica (Statsoft).

\section{Results}

\section{Rimonabant decreases breakpoints}

During baseline and vehicle conditions rats reached breakpoints following an average of $14(\mathrm{SEM}=0.45)$ and $14(\mathrm{SEM}=0.87)$ pellets, respectively. These correspond to an average breakpoint or last response ratio completed during baseline of 78.2 (SEM = 7.39) and during vehicle of 89.8 (SEM = 16.85). Rimonabant 
(150 $\mu \mathrm{g} / \mathrm{kg}$, i.v.) robustly reduced the breakpoint in all animals tested, average breakpoint of $18.4(\mathrm{SEM}=6.30)$, with the corresponding reduction in the number of pellets earned [6.8 (SEM = 1.62)]. Repeated-measures ANOVA showed a significant effect of rimonabant across conditions $\left(F_{(2,10)}=12.47 ; p<0.001\right)$. Post hoc comparisons showed that breakpoints obtained under rimonabant were significantly lower than those obtained during baseline and vehicle (Fig. 1).

NAc cell classification according to waveform characteristics Neurons in the NAc are comprised mostly by MSNs, which constitute $\sim 95 \%$ of the total neurons in this structure (Kemp and Powell, 1971), with the rest of the neuronal population consisting mainly of cholinergic interneurons (Armstrong et al.,1983), persistent low-threshold spiking interneurons (Bevan et al., 1998), and FSIs (Berke et al., 2004; Lansink et al., 2010; Morra et al., 2010). In behaving animals most of the neurons that show a correlation with operant tasks are believed to be MSNs; FSIs do not show a precise spike synchronization with different events in operant tasks (Berke, 2008), but they may control LFP oscillatory power (Berke et al., 2004; Berke, 2005; van der Meer et al., 2010). Given these differential characteristics between MSNs and FSIs it was necessary to distinguish these populations of neurons. A total of 133 (mean number of neurons per rat $=26.6$; $\mathrm{SEM}=4.03$ ) neurons was recorded in the NAc during the PR task; of those neurons 7 (5.26\%) were classified as FSI, 125 (93.98\%) as MSN, and 1 as other $(0.75 \%)$. The characterization of neuronal types was done based on waveform shape (Fig. $2 a$; Table 1); putative FSIs were identified as having a valley FWHM equal or inferior to $150 \mu$ s and a firing rate of $>7.5 \mathrm{~Hz}$. The rest were treated as putative MSNs. The neuron with a valley width of $>500 \mu$ s was classified as other. Representative FSI and MSN waveform shapes are presented in Figure 2, $b$ and $c$, respectively.

\section{NAc cell firing patterns during PR responding}

Nicola and Deadwyler (2000) showed that the firing rate of neurons in the NAc can be broadly classified into LPE and LPI when subjects are working for cocaine under a PR schedule. We aimed to verify if this classification applied for rats working for food pellets and whether these distinctive firing rates encompassed a CB1R-mediated component. Analysis of integrated firing rate histograms identified 21 neurons (16\%) as LPE and 14 neurons (11\%) as LPI, whereas the remaining neurons did not show any recognizable firing patterns during the task, consistent with previous studies (Carelli and Deadwyler, 1994; Carelli et al., 2000). None of the neurons showing LPE or LPI firing patterns were identified as FSI. The firing rates of LPE cells increased gradually across trials with increases in firing before the onset of each bout of lever pressing (Fig. 3). The highest overall firing rate was observed at breakpoint. During vehicle, peak firing rates for LPE neurons averaged $1.99 \mathrm{~Hz}(\mathrm{SEM}=0.59)$; following rimonabant this peak did not significantly change $2.16 \mathrm{~Hz}\left(\mathrm{SEM}=0.78 ; t_{(20)}\right.$ $=-0.61 ; p=0.54)$. In contrast, the firing rates of LPI cells decreased across trials. Specifically, these neurons showed a decrease in activity at the beginning of the session, which was sustained for the duration of responding (Fig. 4). Rimonabant significantly reduced activity before the beginning of the session compared with vehicle $\left(t_{(13)}=-3.42 ; p=0.004\right)$ from 1.43 $(\mathrm{SEM}=0.29)$ to $1.08 \mathrm{~Hz}(\mathrm{SEM}=0.24)$.

\section{Event-specific characteristics of NAc neuron activity}

To analyze time-locked firing patterns within the task, we examined activity at reward delivery and its associated cues and cue
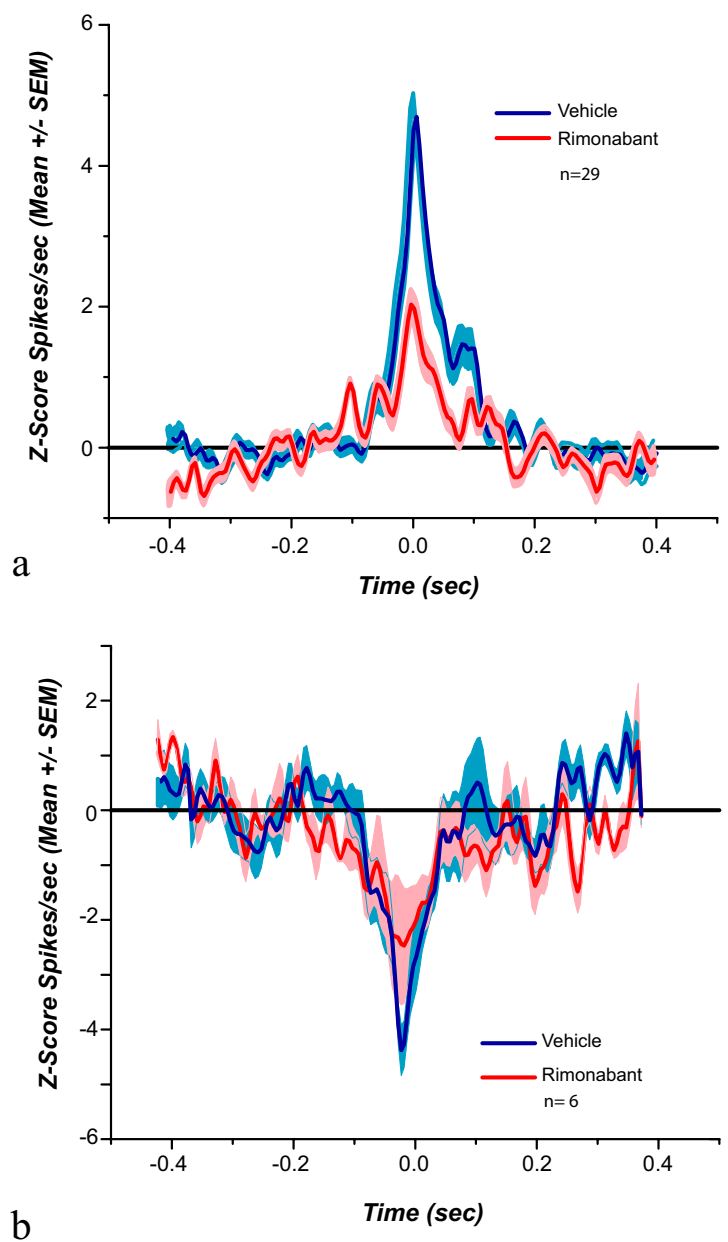

Figure 7. Average $(\boldsymbol{a})$ peak $(n=29)$ and $(\boldsymbol{b})$ trough $(n=6)$ cross-correlations. Rimonabant significantly reduces positive functional cross-correlations observed among neighboring neurons. Shade indicates SEM.

onset at the beginning of each trial. Table 2 shows the percentage of neurons showing different response types to these events. Of the 133 neurons recorded during the task, 78 (58.64\%) exhibited no patterned activity. The remaining 55 cells (41.35\%), all MSNs, exhibited excitatory or inhibitory firing patterns relative to cue presentation or reward delivery. Specifically, 36 (27\%) neurons exhibited patterned activity relative to reward delivery; of these $27(20 \%)$ showed a transient increase in firing rate, $5(3.75 \%)$ showed a sustained increase, and $4(3 \%)$ showed a sustained inhibition. Nineteen (14.3\%) neurons exhibited patterned activity relative to the cue presentation: $10(7.5 \%)$ showed a transient increase in firing rate, $4(3 \%)$ showed a sustained increase, and 5 $(3.75 \%)$ showed a sustained inhibition.

\section{CB1 receptor blockade modifies neural encoding associated with reward delivery}

A $3 \mathrm{D}$ representation of the perievent rasters of representative neurons responsive to reward delivery and its associated cues following vehicle and rimonabant administration, along with the normalized population activity graph, are shown in Figure 5. Neural activity is depicted $8 \mathrm{~s}$ before and after reward delivery, which is set at 0 across all trials. The translucent rectangle marks the injection of rimonabant. Figure $5 a$ depicts a representative neuron that showed a transient increase in firing during vehicle at 
reward delivery, and a profound decrease in this transient activity was observed following rimonabant. The decrease in transient activity is evident when the mean normalized firing rate from the 27 neurons that displayed phasic excitation upon reward delivery is plotted (Fig. $5 b$ ). $\mathrm{Ri}-$ monabant significantly attenuated the peak observed when contrasted against vehicle $\left(t_{(26)}=3.510 ; p=0.0008\right)$.

Figure $5 c$ shows a representative neuron that showed a sustained increase in firing rate after reward delivery and its associated cues and the effect of rimonabant in this particular type of patterned discharge (Fig. $5 d$ ). Paired $t$ tests performed for the collapsed data before and after the reward delivery show that there is a significant difference before and after the reward when the firing rate is recorded under vehicle $\left(t_{(4)}=-9.460 ; p=0.0006\right)$, which remains unchanged following rimonabant $\left(t_{(4)}=-2.262 ; p=0.086\right)$. However, when firing rates after reward delivery are compared, a statistical difference $\left(t_{(4)}=10.441 ; p=0.0004\right)$ is observed, confirming that rimonabant significantly reduced the postreinforcement excitation.

Finally, a subset of neurons showed a time-locked decrease in firing at reward delivery and its associated cues. In these neurons rimonabant failed to significantly alter the observed decrease (Fig. 5e shows a representative neuron). Firing rate after reward delivery for this population of cell was not significantly different between vehicle and rimonabant (Fig. $5 f ; t_{(3)}=-2.177 ; p=0.117$ ).

\section{CB1 receptor blockade modifies neural encoding associated with the cue that indicates trial start}

The $3 \mathrm{D}$ representation of the perievent rasters of representative neurons responsive to the cue that indicates the beginning of the trial during vehicle and rimonabant treatment, along with a normalized $Z$ score of population neural activity graph, is shown in Figure 6. As before, the translucent rectangle in the 3D graphs marks the injection of rimonabant. Figure $6 a$ shows a representative neuron displaying a brief cue-evoked increase in firing at the cue. CB1R blockade significantly attenuated this pattern compared with vehicle (Fig. $6 b ; t_{(9)}=6.010 ; p=0.0002$ ).

CB1R blockade also disrupted neurons showing a sustained increase in firing rate observed after cue onset (Fig. $6 c, d ; t_{(3)}=$ 3.365; $p=0.0435$ ). This significant effect indicates that the sustained firing rate obtained under rimonabant is lower than that observed following vehicle administration. Last, rimonabant treatment altered the firing rate decrease observed after cue onset compared with vehicle; such that there was no differentiation in firing activity before or after cue onset $\left(t_{(4)}=\right.$ $-1.310 ; p=0.130)$. Such effect is evident in the representative example (Fig. 6e) as well as in the mean normalized firing rate graph for the pooled population data $\left(t_{(4)}=-13.4737 ; p=\right.$ 0.0001; Fig. 6f).
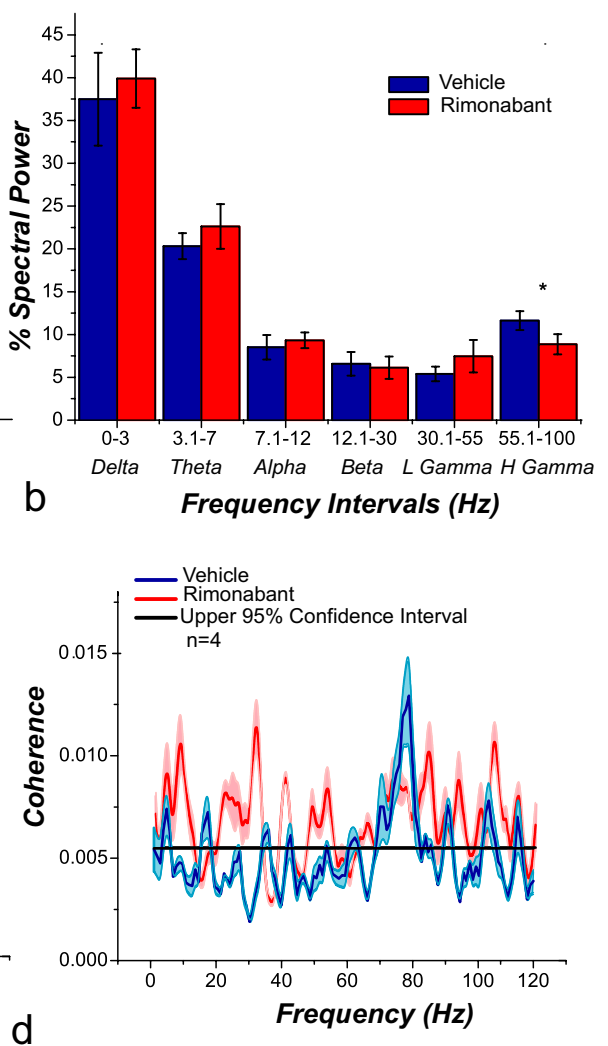

d

g the PR task after vehicle and rimonabant administration $(n=6)$. Under the influence of rimonabant there is a change in the frequency at which the power peaks and a change in power in the gamma band. $\boldsymbol{b}$, Bar graph depicting weight of the spectral bands during the task. Rimonabant significantly reduces the observed weight on high gamma power $(55-100 \mathrm{~Hz})$. Spike-field coherence for FSIs. A group of these neurons $(n=3)$ preferentially change their firing rate during gamma-50 (c), whereas the remaining $(n=4)$ do so during gamma-80 (d). Rimonabant administration produces an increase in the frequency range at which these neurons were entrained.

\section{Collective synchrony in NAc neurons is affected} by rimonabant

Functional connectivity among simultaneously recorded neurons was analyzed via pairwise cross-correlations (Fig. 7). For each subject, each of the recorded neurons was used as a reference and cross-correlated with the rest of the neurons (all cell-pair combinations were analyzed). During vehicle there were 29 crosscorrelograms with a peak (mean $Z$ score peak $=4.85 ; \mathrm{SEM}=0.57$ ) and 6 with a trough at time 0 (mean $Z$ score trough $=-4.38$; SEM $=$ 0.42). Following rimonabant administration there was a significant reduction in the peak of the population cross-correlogram (mean $Z$ score peak $=2.54 \mathrm{SEM}=0.37)\left(\right.$ Fig. $\left.7 a ; t_{(28)}=4.55 ; p=0.0001\right)$. A reduction in the population trough was also observed following rimonabant (mean $Z$ score trough $=-2.43 \mathrm{SEM}=1.031$; Fig. $7 b$ ) but was not statistically reliable $\left(t_{(5)}=0.73 ; p=0.49\right)$. None of the cross-correlograms analyzed that used FSI neurons as a reference yielded significant cross-correlations with either MSNs or other FSIs.

\section{Accumbal LFP activity in the gamma range is disrupted following CB1 receptor blockade}

LFP oscillations are believed to reflect the organization and synchronized activity between different brain areas related to different cognitive and motor processes (Sanes and Donoghue, 1993; Buzsáki, 2006). Here we measured LFPs with the same electrodes used to record action potentials. We constructed session-wide spectrograms to analyze changes in LFPs before and after the administration of rimonabant (Fig. 8a). Rimonabant changed the frequency at which gamma power peaked and attenuated 


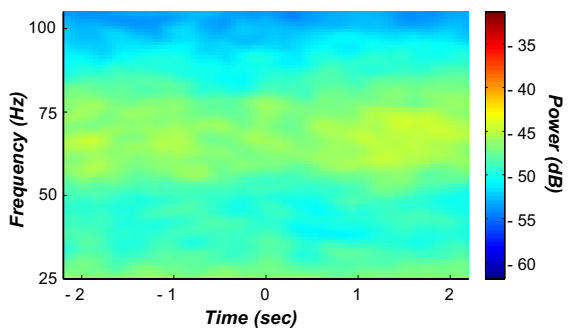

a
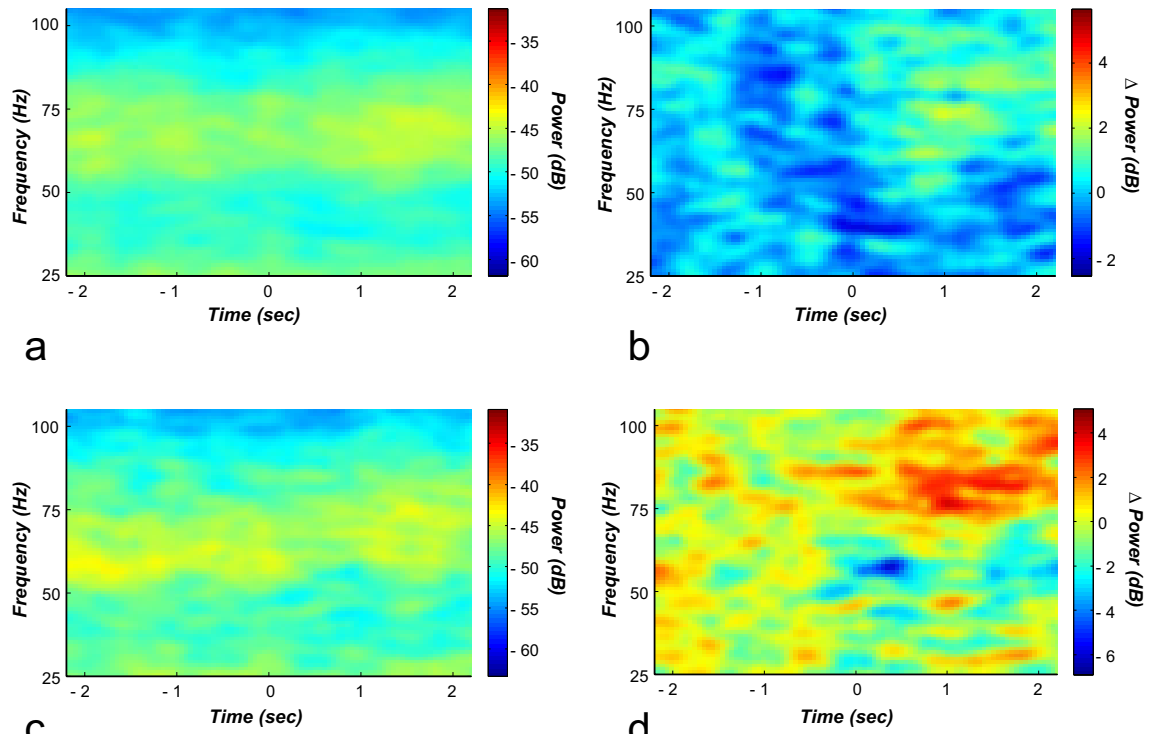

b
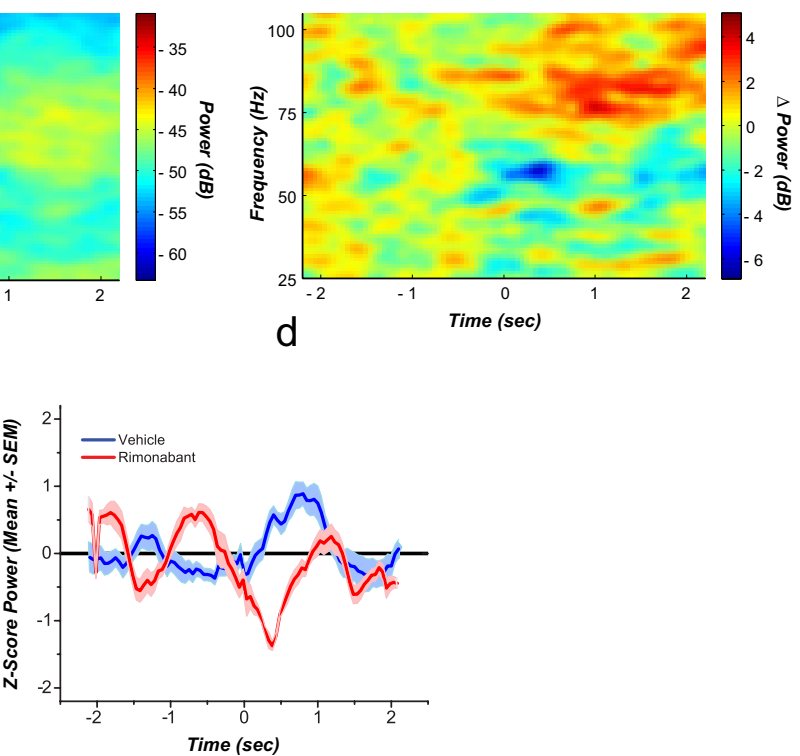

e

Figure 9. Perievent spectrogram for cue onset. The spectrogram is aligned to the cue that indicates the beginning of the trial at time 0. $\boldsymbol{a}$, Shows that after vehicle administration there is not an evident time-locked change in the power of the different spectral bands. This observation is confirmed in the baseline subtracted spectrogram (b). After rimonabant administration a slight increase in the low gamma band, particularly before the cue onset, is observed (c). The baseline subtracted spectrogram shows that there is a time-locked decrease around gamma-55 (d). e, Mean gamma-50 power z scores $(n=6)$; in this graph the time-locked reduction in gamma- 50 is evident.

overall power in the gamma band. Specifically, the peak of the gamma band shifted from $64.11 \mathrm{~Hz}(\mathrm{SEM}=2.38)$ during vehicle to $55.84 \mathrm{~Hz}(\mathrm{SEM}=1.49)$ following rimonabant $\left(t_{(4)}=2.99 ; p=\right.$ $0.0402)$. Figure $8 b$ shows the distribution of power across all spectral bandwidths during the task obtained after vehicle (blue) and rimonabant (red) administration. The only significant changes were observed in the gamma bandwidth $\left(55-100 \mathrm{~Hz} ; t_{(4)}=2.93\right.$; $p=0.0424)$.

Gamma oscillations are hypothesized to couple to FSIs in the striatum (Berke, 2005; van der Meer and Redish, 2009). We analyzed these neurons to calculate spike-field coherence to determine whether LFPs were partially affected by the CB1R antagonist. We found, as previously reported (Berke, 2009; van der Meer and Redish, 2009), that there were two groups of FSI neurons, one of which changed their firing rate differentially with "gamma-50" (45-55 Hz) and the other with "gamma-80" $(70-85 \mathrm{~Hz})$ powers (Fig. $8 c, d$ ). Following rimonabant administration, FSIs lost their synchronous firing to these frequency bands and became coherent to multiple frequencies.

Gamma oscillations are differentially modulated by rimonabant during cue presentation and reward delivery Perievent spectrograms showing the frequencies of the low and high gamma spectral bands were constructed around cue presentation
(Fig. $9 a-d$ ) and reward delivery (Fig. 10ad). The different bands were studied at cue onset for either vehicle (Fig. 9a) or rimonabant (Fig. 9c). Baseline-subtracted spectrograms depict time-locked changes in power at the different spectral bands during vehicle (Fig. 9b) or rimonabant (Fig. 9d). Mean gamma-50 $(45-55 \mathrm{~Hz})$ power $Z$ scores increased slightly following cue onset during vehicle. Under rimonabant this increase disappears and instead a trough in gamma-50 power is observed (Fig. 9e). This decrease is confirmed when the $Z$ values of the troughs observed after cue onset, from the baseline subtracted powers, are contrasted $\left(t_{(4)}=\right.$ $3.34 ; p=0.002)$.

Figure $10 a$ depicts spectrograms obtained during vehicle administration at reward delivery and its associated cues. A significant increase in power is observed around high gamma immediately following reward delivery $\left(t_{(4)}=3.20, p=0.03\right.$; Fig. 10b). Rimonabant administration (Fig. 10c) significantly reduced high gamma after reward delivery and its associated cues $\left(t_{(4)}=\right.$ 2.31, $p=0.02$; Fig. $10 d)$. Mean gamma- 80 (70-85 Hz) power $Z$ scores observed are transiently elevated following vehicle and this increase is completely abolished by rimonabant administration (Fig. 10e). Analysis of gamma power peaks, obtained from the baseline subtracted powers, confirms a highly significant statistical difference between vehicle and rimonabant $\left(t_{(4)}=3.80\right.$; $p=0.01$ ).

\section{Discussion}

The present research replicates and extends knowledge from previous studies showing that blockade of CB1Rs potently reduce breakpoints in the PR schedule (Ward and Dykstra, 2005; Maccioni et al., 2008). We further find that this behavioral effect is accompanied by profound changes in neural encoding related to food delivery, by its associated cues, and by predictive cue onset in the NAc.

\section{Effects of rimonabant on general cell firing in the NAc}

Our results confirm previous categorizations of session-wide NAc activity during PR (LPE or LPI cells) (Nicola and Deadwyler, 2000). The reduction in breakpoints observed following CB1R blockade was accompanied by a modest effect on LPE cells and a reduction of the initial peak of LPI cells. Nicola and Deadwyler (2000) suggested that the increase in firing rate observed in LPE neurons is correlated with the abolishment of the operant response. Their interpretation fits our data in that the breakpoint was followed by the highest increase in firing rate during which responding was absent. If the firing increase leads to response cessation, then rimonabant should not affect this firing increase as our data demonstrate. Nicola and Deadwyler (2000) proposed that the firing rate increase observed in LPE neurons is controlled by DA release within the NAc due to fluctuating intracerebral cocaine concentrations. However, our recordings suggest that LPE patterns can emerge in the absence of cocaine. The intrinsic 
increase in the inter-reward interval brought by the PR schedule may be sufficient to produce an increase of DA levels as has recently been shown in voltammetric experiments (Wanat et al., 2010); this would allow LPE cells to change their activity and ultimately lead to decreased responding. It is likely that the decrease in breakpoint observed was in part a product of the inhibitory effect that CB1R blockade has over DA release (Cheer et al., 2007; Melis et al., 2007; Trujillo-Pisanty et al., 2011, Oleson et al., 2012). CB1R antagonists can decrease DA release by affecting the binding of eCBs to CB1Rs present on glutamatergic terminals located in several nuclei that send glutamatergic projections to the VTA (Geisler et al. 2007), and control its bursting activity (Riegel and Lupica, 2004) or by interfering with eCBs binding to presynaptic CB1Rs on GABA terminals in the VTA (Cohen et al., 2002; Szabo et al., 2002; Lupica and Riegel, 2005). The significant reduction observed in the initial peak on LPI cells after rimonabant administration could underlie the decreased reward density that is observed at the beginning of the session, and this could be tied to a reduction of DA release following reward delivery.

\section{Effects of rimonabant on neural encoding in the Nac}

We observed patterned activity to specific events in the task (reward delivery and the cue that signals the beginning of the trial) as previously reported (Nicola and Deadwyler, 2000). In particular, transient and long-lasting excitations as well as inhibitions that coincided with reward delivery and cue onset were observed. Such patterns of activation have been described before in rodents performing different appetite-motivated tasks (Hollander et al., 2002; Nicola et al., 2004a,b, Roesch et al., 2009; Cacciapaglia et al., 2011). The neural excitation observed following reward delivery is believed to encode the unconditional properties of the reward; in particular short phasic responses have been associated with reward characteristics such as palatability and magnitude (Cromwell and Schultz, 2003; Taha and Fields, 2005). Functionally, this excitation has been suggested to provide information about the motivational significance of reward to other circuits (Apicella et al., 1991). On the other hand, it has been suggested that the inhibition observed during reward delivery facilitates disinhibition of target neurons in motor areas related to consummatory behaviors (Nicola et al., 2004b; Roitman et al., 2005; Taha and Fields, 2006; Krause et al., 2010). The firing changes correlated with cue presentation have also been linked to different functional aspects of reward-directed behavior. The transient increase in firing rate observed with cue onset is hypothesized to encode the learned significance of the reward and the potential consequences of the behavior (Hassani et al., 2001; Cromwell and Schultz, 2003; Roesch et al., 2009). Therefore, these neurons are thought to encode incentive information (Nicola et al., 2004a; Smith et al.,

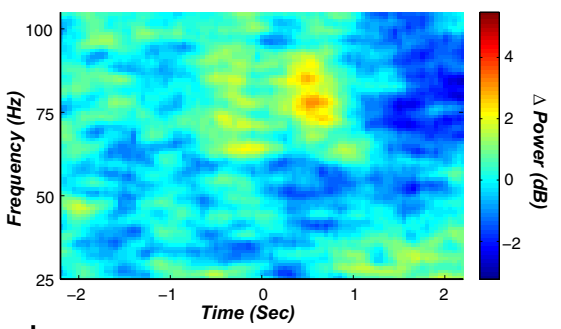

b
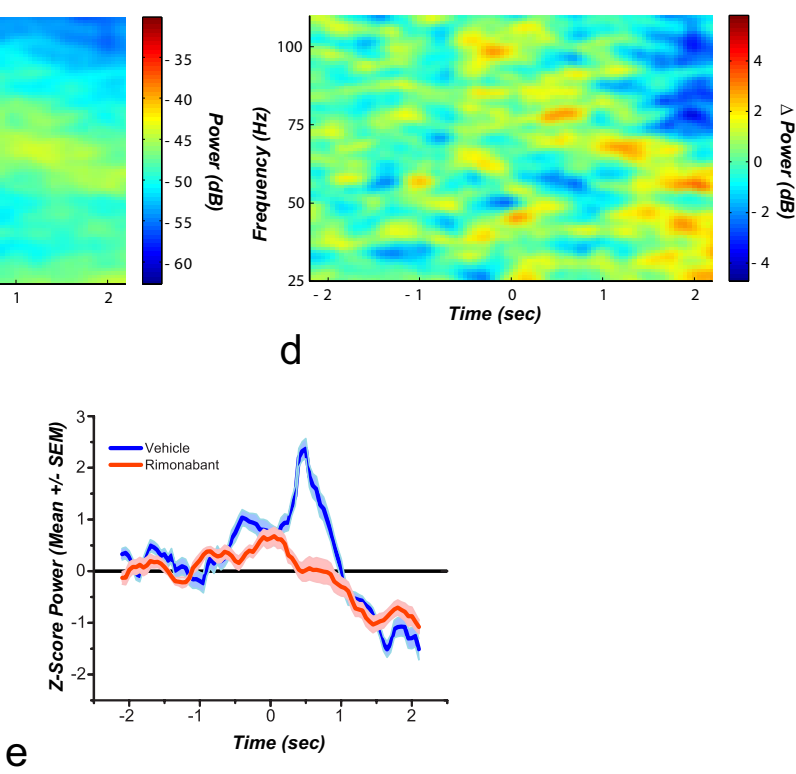

e d

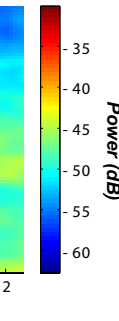

\section{.}

Figure 10. Perievent spectrogram for reward delivery and its associated cues. The spectrogram is aligned to reward delivery at time $0 . \boldsymbol{a}$, Shows that after vehicle administration there is an increase in gamma-80 power immediately after reward delivery, and the baseline subtracted spectrogram $(\boldsymbol{b})$. After rimonabant administration this locked increase in is appears $(\boldsymbol{c})$. This change is evident in the baseline subtracted spectrogram (d). $\boldsymbol{e}$, Mean gamma-80 power z scores $(n=6)$. In this graph the time-locked increase in gamma- 80 with reward delivery observed after vehicle administration is absent following the blockade of $C B 1$ receptors.

2011). Sustained increases in firing rate have been suggested to serve in maintaining a representation of the anticipated reward (Roesch et al., 2009). Time-locked inhibitions, such as those reported here, have been proposed to gate goal-directed behaviors. Specifically, the activity of a subpopulation of NAc neurons is believed to tonically inhibit behavior and suppression of activity in this particular population is necessary for operant behavior to occur (Taha and Fields, 2006; Krause et al., 2010).

Blockade of CB1Rs produced profound changes in the firing patterns associated with each of these functional roles. Rimonabant decreased the excitation peaks related to the unconditional properties of the reward as well as of the magnitude of peaks related to incentive salience. It also affected neural activation related to the representation of the anticipated reward, and produced a change in inhibitory signaling related to aspects of consummatory and reward directed-behavior. The blockade of eCB signaling by rimonabant was not restricted to individual neural firing patterns. It significantly weakened the connection strength between neighboring neurons within the NAc, specifically those showing positive cross-correlations. The reduction in the strength of functional connectivity could underlie the alteration of firing patterns, probably through an increase in neural noise among NAc ensembles. The lack of negative cross-correlation between FSI and MSNs has been previously reported (Berke, 
2009), and this could mean that not all recorded neurons are connected (alternatively this may be a consequence of our limited dataset for FSIs).

\section{Effects of rimonabant on neural rhythmicity}

Gamma oscillations are believed to serve as temporal structures that allow the alignment of spike trains within and between brain areas (Buzsáki, 2006; Kalenscher et al., 2010) and thus facilitate the integration of different afferents into NAc firing patterns (Kalenscher et al., 2010). LFPs can be the product of electrical field changes produced in other brain areas rather than the one in which the electrodes are located (Berke, 2005). However, and in accordance with prior studies, our data show that LFPs recorded from the NAc are in part controlled by FSIs (Berke, 2005; van der Meer and Redish, 2009; Kalenscher et al., 2010, Morra et al., 2012). Gamma-80 has been previously linked to reward delivery (Berke, 2004; van der Meer and Redish, 2009), whereas gamma-50 has been linked among other functions to movement initiation (Masimore et al., 2004; for a review, see van der Meer et al., 2010). CB1R blockade altered the synchronicity between FSIs and these bands, expanding the range of frequencies at which they entrained. Consequently, the communication bridge among different brain structures and neural ensembles that were coordinated by these gamma bands and possibly facilitated the organization and control of the behavior was disrupted. Evidence of this disruption is evident when rimonabant, along with the reduction in breakpoints, produced a decrease in gamma- 80 that was coincident upon reward delivery and a decrease in gamma-50 that was coincident with the cue signaling the start of the trial.

The changes in activation patterns and connection strength shown here reveal the heterogeneity of neural ensembles that are contained in the NAc, and shed light on the surprising uniformity of effects that the blockade of CB1Rs has on those ensembles. eCBs work as retrograde messengers and contribute to short- and long-term modulation of synaptic transmission via presynaptic mechanisms. Blockade of CB1Rs could have affected neural encoding via perturbations of glutamatergic or GABAergic signaling. Glutamatergic afferents to the NAc, including the amygdala, the cortex, and the hippocampus, may have compromised glutamatergic transmission at the synapse at NAc when responding occurred under rimonabant (Robbe et al., 2002; Domenici et al., 2006). Likewise, blockade of CB1Rs could have prevented eCBmediated reduction of GABA release in the NAc (Hoffman and Lupica, 2001). The reduction of inhibition in GABAergic neurotransmission by eCBs is believed to facilitate the responsiveness to excitatory cortical inputs and increase NAc output pathways (Hoffman and Lupica, 2001). The interference of eCB signaling by rimonabant would have probably interfered with this inhibitory mechanism, making NAc neurons less responsive to cortical inputs. Indirectly the blockade of CB1 signaling on excitatory and inhibitory synapses alters the excitability of DA neurons in the VTA and DA release in the NAc, which in turn affects the synaptic efficacy of MSNs (O’Donnell and Grace, 1995, 1998). Accordingly, previous research has shown that inactivation of VTA DA cell bodies or injections of D1 or D2 receptor antagonists into the NAc significantly reduced the peak and inhibition firing patterns associated with incentive cues (Yun et al., 2004; Cheer et al., 2007) as well as rewards (Cacciapaglia et al., 2011).

The present results demonstrate that the eCB system plays a fundamental role in the encoding of reward and incentive cues at the level of the NAc by allowing for neural synchrony and rhythmicity patterns to emerge during reinforcement processing. Furthermore, our findings suggest that eCBs are a highly conserved regulatory system that fine-tunes the function of limbic and motor inputs to organize motivated behaviors.

\section{References}

Alger BE (2002) Retrograde signaling in the regulation of synaptic transmission: focus on endocannabinoids. Prog Neurobiol 68:247-286.

Apicella P, Ljungberg T, Scarnati E, Schultz W (1991) Responses to reward in monkey dorsal and ventral striatum. Exp Brain Res 85:491-500.

Armstrong DM, Saper CB, Levey AI, Wainer BH, Terry RD (1983) Distribution of cholinergic neurons in the rat brain demonstrated by the immunocytochemical localisation of choline acetyltransferase. J Comp Neurol 216:53-68.

Berke JD (2005) Participation of striatal neurons in large-scale oscillatory networks. In: The basal ganglia VIII (Bolam JP, Ingham CA, Magill PJ, eds), pp 25-35. New York: Springer.

Berke JD (2008) Uncoordinated firing rate changes of striatal fastspiking interneurons during behavioral task performance. J Neurosci 28:10075-10080.

Berke JD (2009) Fast oscillations in cortical-striatal networks switch frequency following rewarding events and stimulant drugs. Eur J Neurosci 30:848-859.

Berke JD, Okatan M, Skurski J, Eichenbaum HB (2004) Oscillatory entrainment of striatal neurons in freely moving rats. Neuron 43:883-896.

Berridge KC (2007) The debate over dopamine's role in reward: the case for incentive salience. Psychopharmacology 191:391-431.

Bevan MD, Booth PA, Eaton SA, Bolam JP (1998) Selective innervation of neostriatal interneurons by a subclass of neuron in the globus pallidus of the rat. J Neurosci 18:9438-9452.

Bidaut-Russell M, Devane WA, Howlett AC (1990) Cannabinoid receptors and modulation of cyclic AMP accumulation in the rat brain. J Neurochem 55:21-26.

Burkhardt JM, Jin X, Costa RM (2009) Dissociable effects of dopamine on neuronal firing rate and synchrony in the dorsal striatum. Front Integr Neurosci 3:28.

Buzsáki G (2006) Rhythms of the brain. Oxford, UK: Oxford UP.

Cacciapaglia F, Wightman RM, Carelli RM (2011) Rapid dopamine signaling differentially modulates distinct microcircuits within the nucleus accumbens during sucrose-directed behavior. J Neurosci 31:13860-13869.

Cardinal RN, Parkinson JA, Hall J, Everitt BJ (2002) Emotion and motivation. the role of the amygdala, ventral striatum, and prefrontal cortex. Neurosci Biobehav Rev 26:321-352.

Carelli RM, Deadwyler SA (1994) A comparison of nucleus accumbens neuronal firing patterns during cocaine self-administration and water reinforcement in rats. J Neurosci 14:7735-7746.

Carelli RM, Ijames SG, Crumling AJ (2000) Evidence that separate neural circuits in the nucleus accumbens encode cocaine versus "natural" (water and food) reward. J Neurosci 20:4255-4266.

Cheer JF, Marsden CA, Kendall DA, Mason R (2000) Lack of response suppression follows repeated ventral tegmental cannabinoid administration: an in vitro electrophysiological study. Neuroscience 99:661-667.

Cheer JF, Kendall DA, Mason R, Marsden CA (2003) Differential cannabinoid-induced electrophysiological effects in rat ventral tegmentum. Neuropharmacology 44:633-641.

Cheer JF, Aragona BJ, Heien ML, Seipel AT, Carelli RM, Wightman RM (2007) Coordinated accumbal dopamine release and neural activity drive goal-directed behavior. Neuron 54:237-244.

Cippitelli A, Bilbao A, Hansson AC, del Arco I, Sommer W, Heilig M, Massi M, Bermúdez-Silva FJ, Navarro M, Ciccocioppo R, de Fonseca FR de (2005) Cannabinoid CB1 receptor antagonism reduces conditioned reinstatement of ethanol-seeking behavior in rats. Eur J Neurosci 21:2243-2251.

Cohen C, Perrault G, Voltz C, Steinberg R, Soubrié P (2002) SR141716, a central cannabinoid $(\mathrm{CB}(1))$ receptor antagonist, blocks the motivational and dopamine-releasing effects of nicotine in rats. Behav Pharmacol 13:451-463.

Cota D, Marsicano G, Lutz B, Vicennati V, Stalla GK, Pasquali R, Pagotto U (2003) Endogenous cannabinoid system as a modulator of food intake. Int J Obes Relat Metab Disord 27:289-301.

Cromwell HC, Schultz W (2003) Effects of expectations for different reward magnitudes on neuronal activity in primate striatum. J Neurophysiol 89:2823-2838.

De Vry J, Schreiber R, Eckel G, Jentzsch KR (2004) Behavioral mechanisms 
underlying inhibition of food-maintained responding by the cannabinoid receptor antagonist/inverse agonist SR141716A. Eur J Pharmacol 483:55-63.

Domenici MR, (2006) Cannabinoid receptor type 1 located on presynaptic terminals of principal neurons in the forebrain controls glutamatergic synaptic transmission. J Neurosci 26:5794-5799.

Efron B (1979) Bootstrap methods: another look at the jackknife. Ann Stat $7: 1-26$.

Filip M, Gołda A, Zaniewska M, McCreary AC, Nowak E, Kolasiewicz W, Przegaliński E (2006) Involvement of cannabinoid CB1 receptors in drug addiction: effects of rimonabant on behavioral responses induced by cocaine. Pharmacol Rep 58:806-819.

Gallate JE, Saharov T, Mallet PE, McGregor IS (1999) Increased motivation for beer in rats following administration of a cannabinoid CB1 receptor agonist. Eur J Pharmacol 370:233-240.

Geisler S, Derst C, Veh RW, Zahm DS (2007) Glutamatergic afferents of the ventral tegmental area in the rat. J Neurosci 27:5730-5743.

Hassani OK, Cromwell HC, Schultz W (2001) Influence of expectation of different rewards on behavior-related neuronal activity in the striatum. J Neurophysiol 85:2477-2489.

Henry DJ, Chavkin C (1995) Activation of inwardly rectifying potassium channels (GIRK1) by co-expressed rat brain cannabinoid receptors in Xenopus oocytes. Neurosci Lett 186:91-94.

Higgs S, Barber DJ, Cooper AJ, Terry P (2005) Differential effects of two cannabinoid receptor agonists on progressive ratio responding for food and free-feeding in rats. Behav Pharmacol 16:389-393.

Hodos W (1961) Progressive ratio as a measure of reward strength. Science 134:943-944.

Hoffman AF, Lupica CR (2000) Mechanisms of cannabinoid inhibition of GABA(A) synaptic transmission in the hippocampus. J Neurosci 20:2470-2479.

Hoffman AF, Lupica CR (2001) Direct actions of cannabinoids on synaptic transmission in the nucleus accumbens: a comparison with opioids. J Neurophysiol 85:72-83.

Hollander JA, Ijames SG, Roop RG, Carelli RM (2002) An examination of nucleus accumbens cell firing during extinction and reinstatement of water reinforcement behavior in rats. Brain Res 929:226-235.

Kalenscher T, Lansink CS, Lankelma JV, Pennartz CM (2010) Rewardassociated gamma oscillations in ventral striatum are regionally differentiated and modulate local firing activity. J Neurophysiol 103:1658-1672.

Kemp JM, Powell TP (1971) The connexions of the striatum and globus pallidus. Synthesis and speculation. Philos Trans R Soc London B Biol Sci 262:441-457.

Kim H, Sul JH, Huh N, Lee D, Jung MW (2009) Role of striatum in updating values of chosen actions. J Neurosci 29:14701-14712.

Krause M, German PW, Taha SA, Fields HL (2010) A pause in nucleus accumbens neuron firing is required to initiate and maintain feeding. J Neurosci 30:4746-4756.

Lansink CS, Goltstein PM, Lankelma JV, Pennartz CM (2010) Fast-spiking interneurons of the rat ventral striatum: temporal coordination of activity with principal cells and responsiveness to reward. Eur J Neurosci 32:494-508.

Le Foll B, Goldberg SR (2005) Cannabinoid CB1 receptor antagonists as promising new medications for drug dependence. J Pharmacol Exp Ther 312:875-883.

Lidierth M (2009) sigTOOL: a MATLAB-based environment for sharing laboratory-developed software to analyze biological signals. J Neurosci Methods 178:188-196.

Lupica CR, Riegel AC (2005) Endocannabinoid release from midbrain dopamine neurons: a potential substrate for cannabinoid receptor antagonist treatment of addiction. Neuropharmacology 48:1105-1116.

Maccioni P, Pes D, Carai MA, Gessa GL, Colombo G (2008) Suppression by the cannabinoid CB1 receptor antagonist, rimonabant, of the reinforcing and motivational properties of a chocolate-flavoured beverage in rats. Behav Pharmacol 19:197-209.

Masimore B, Kakalios J, Redish AD (2004) Measuring fundamental frequencies in local field potentials. J Neurosci Methods 138:97-105.

Melis M, Pistis M, Perra S, Muntoni AL, Pillolla G, Gessa GL (2004) Endocannabinoids mediate presynaptic inhibition of glutamatergic transmission in rat ventral tegmental area dopamine neurons through activation of CB1 receptors. J Neurosci 24:53-62.

Melis T, Succu S, Sanna F, Boi A, Argiolas A, Melis MR (2007) The canna- binoid antagonist SR 141716A (Rimonabant) reduces the increase of extra-cellular dopamine release in the rat nucleus accumbens induced by a novel high palatable food. Neurosci Lett 419:231-235.

Mogenson GJ, Jones DL, Yim CY (1980) From motivation to action. Functional interface between the limbic system and the motor system. Prog Neurobiol 14:69-97.

Morra JT, Glick SD, Cheer JF (2012) Cannabinoid receptors mediate methamphetamine induction of high frequency gamma oscillations in the nucleus accumbens. Neuropharmacology 63:565-574.

Morra JT, Glick SD, Cheer JF (2010) Neural encoding of psychomotor activation in the nucleus accumbens core, but not the shell, requires cannabinoid receptor signaling. J Neurosci 30:5102-5107.

Nicola SM, Deadwyler SA (2000) Firing rate of nucleus accumbens neurons is dopamine-dependent and reflects the timing of cocaine-seeking behavior in rats on a progressive ratio schedule of reinforcement. J Neurosci 20:5526-5537.

Nicola SM, Surmeier J, Malenka RC (2000) Dopaminergic modulation of neuronal excitability in the striatum and nucleus accumbens. Annu Rev Neurosci 23:185-215.

Nicola SM, Yun IA, Wakabayashi KT, Fields HL (2004a) Cue-evoked firing of nucleus accumbens neurons encodes motivational significance during a discriminative stimulus task. J Neurophysiol 91:1840-1865.

Nicola SM, Yun IA, Wakabayashi KT, Fields HL (2004b) Firing of nucleus accumbens neurons during the consummatory phase of a discriminative stimulus task depends on previous reward predictive cues. J Neurophysiol 91:1866-1882.

O'Donnell P, Grace AA (1995) Synaptic interactions among excitatory afferents to nucleus accumbens neurons. hippocampal gating of prefrontal cortical input. J Neurosci 15:3622-3639.

O'Donnell P, Grace AA (1998) Phencyclidine interferes with the hippocampal gating of nucleus accumbens neuronal activity in vivo. Neuroscience 87:823-830.

Oleson EB, Beckert MV, Morra JT, Lansink CS, Cachope R, Abdullah RA, Loriaux AL, Schetters D, Pattij T, Roitman MF, Lichtman AH, Cheer JF (2012) Endocannabinoids shape accumbal encoding of cue-motivated behavior via CB1 receptor activation in the ventral tegmentum. Neuron 73:360-373.

Pesaran B, Pezaris JS, Sahani M, Mitra PP, Andersen RA (2002) Temporal structure in neuronal activity during working memory in macaque parietal cortex. Nat Neurosci 5:805-811.

Rasmussen EB, Huskinson SL (2008) Effects of rimonabant on behavior maintained by progressive ratio schedules of sucrose reinforcement in obese Zucker (fa/fa) rats. Behav Pharmacol 19:735-742.

Riegel AC, Lupica CR (2004) Independent presynaptic and postsynaptic mechanisms regulate endocannabinoid signaling at multiple synapses in the ventral tegmental area. J Neurosci 24:11070-11078.

Robbe D, Kopf M, Remaury A, Bockaert J, Manzoni OJ (2002) Endogenous cannabinoids mediate long-term synaptic depression in the nucleus accumbens. Proc Natl Acad Sci U S A 99:8384-8388.

Roesch MR, Singh T, Brown PL, Mullins SE, Schoenbaum G (2009) Ventral striatal neurons encode the value of the chosen action in rats deciding between differently delayed or sized rewards. J Neurosci 29:13365-13376.

Roitman MF, Wheeler RA, Carelli RM (2005) Nucleus accumbens neurons are innately tuned for rewarding and aversive taste stimuli, encode their predictors, and are linked to motor output. Neuron 45:587-597.

Salamone JD, Correa M (2002) Motivational views of reinforcement. implications for understanding the behavioral functions of nucleus accumbens dopamine. Behav Brain Res 137:3-25.

Samejima K, Ueda Y, Doya K, Kimura M (2005) Representation of actionspecific reward values in the striatum. Science 310:1337-1340.

Sanes JN, Donoghue JP (1993) Oscillations in local field potentials of the primate motor cortex during voluntary movement. Proc Natl Acad Sci U S A 90:4470-4474.

Shoaib M (2008) The cannabinoid antagonist AM251 attenuates nicotine self-administration and nicotine-seeking behaviour in rats. Neuropharmacology 54:438-444.

Smith KS, Berridge KC, Aldridge JW (2011) Disentangling pleasure from incentive salience and learning signals in brain reward circuitry. Proc Natl Acad Sci U S A 108:E255-E264.

Solinas M, Goldberg SR (2005) Motivational effects of cannabinoids and opioids on food reinforcement depend on simultaneous activation of cannabinoid and opioid systems. Neuropsychopharmacology 30:2035-2045. 
Solinas M, Panlilio LV, Tanda G, Makriyannis A, Matthews SA, Goldberg SR (2005) Cannabinoid agonists but not inhibitors of endogenous cannabinoid transport or metabolism enhance the reinforcing efficacy of heroin in rats. Neuropsychopharmacology 30:2046-2057.

Soria G, Mendizábal V, Touriño C, Robledo P, Ledent C, Parmentier M, Maldonado R, Valverde O (2005) Lack of CB1 cannabinoid receptor impairs cocaine self-administration. Neuropsychopharmacology 30:1670-1680.

Szabo B, Siemes S, Wallmichrath I (2002) Inhibition of GABAergic neurotransmission in the ventral tegmental area by cannabinoids. Eur J Neurosci 15:2057-2061.

Taha SA, Fields HL (2005) Encoding of palatability and appetitive behaviors by distinct neuronal populations in the nucleus accumbens. J Neurosci 25:1193-1202.

Taha SA, Fields HL (2006) Inhibitions of nucleus accumbens neurons encode a gating signal for reward-directed behavior. J Neurosci 26:217-222.

Trujillo-Pisanty I, Hernandez G, Moreau-Debord I, Cossette MP, Conover K, Cheer JF, Shizgal P (2011) Cannabinoid receptor blockade reduces the opportunity cost at which rats maintain operant performance for rewarding brain stimulation. J Neurosci 31:5426-5435.

Twitchell W, Brown S, Mackie K (1997) Cannabinoids inhibit N- and P/Qtype calcium channels in cultured rat hippocampal neurons. J Neurophysiol 78:43-50.

Valjent E, Mitchell JM, Besson MJ, Caboche J, Maldonado R (2002) Behavioural and biochemical evidence for interactions between Delta 9-tetrahydrocannabinol and nicotine. Br J Pharmacol 135:564-578.

van der Meer MA, Redish AD (2009) Low and high gamma oscillations in rat ventral striatum have distinct relationships to behavior, reward, and spiking activity on a learned spatial decision task. Front Integr Neurosci 3:9.

van der Meer MA, Kalenscher T, Lansink CS, Pennartz CM, Berke JD, Redish $\mathrm{AD}$ (2010) Integrating early results on ventral striatal gamma oscillations in the rat. Front Neurosci 4:300.

Verty AN, McGregor IS, Mallet PE (2004b) Consumption of high carbohydrate, high fat, and normal chow is equally suppressed by a cannabinoid receptor antagonist in non-deprived rats. Neurosci Lett 354:217-220.

Wanat MJ, Kuhnen CM, Phillips PE (2010) Delays conferred by escalating costs modulate dopamine release to rewards but not their predictors. J Neurosci 30:12020-12027.

Ward SJ, Dykstra LA (2005) The role of CB1 receptors in sweet versus fat reinforcement. effect of $\mathrm{CB} 1$ receptor deletion, $\mathrm{CB} 1$ receptor antagonism (SR141716A) and CB1 receptor agonism (CP-55940). Behav Pharmacol 16:381-388.

Wilson RI, Nicoll RA (2001) Endogenous cannabinoids mediate retrograde signalling at hippocampal synapses. Nature 410:588-592.

Xi ZX, Spiller K, Pak AC, Gilbert J, Dillon C, Li X, Peng XQ, Gardner EL (2008) Cannabinoid CB1 receptor antagonists attenuate cocaine's rewarding effects: experiments with self-administration and brainstimulation reward in rats. Neuropsychopharmacology 33:1735-1745.

Yun IA, Wakabayashi KT, Fields HL, Nicola SM (2004) The ventral tegmental area is required for the behavioral and nucleus accumbens neuronal firing responses to incentive cues. J Neurosci 24:2923-2933. 